\title{
ASSESSMENT OF MATERNAL STRESS DURING PREGNANCY
}

\section{Dr Lavanya Priyadharshini. V \\ Dr Vidhya Thirunavukkarasu* \\ Dr S. Punitha} Josephine

\section{BDS}

MSN, PhD, Asst Professor, King Faisal University, KSA *Corresponding Author

MSN, PhD, Vice Principal, Karpaga Vinayaga College of Nursing, Madurandagam, Chennai.

ABSTRACT Background: The maternal stress refers to the amount of hardship that a woman faces during a period of stress. It also refers to a woman's personal reaction to the stressor and can be measured by assessing her emotions and feelings at the time that she was exposed to the stressful event. This maternal stress during pregnancy increases the risk of the child having a range of altered neuro developmental outcomes, the emotions and experiences of a pregnant woman impinge on her developing fetus. The investigator had a view to focus this aspect to identify and analyze the level of stress among the pregnant mothers. Objectives: To identify the prevalence of women with stress during pregnancy, to correlate the life stress factors and emotional stress factors associated with pregnancy, to associate the demographic variables with the life and emotional stress factors, to prepare a module to reduce stress and motivate for healthier and happier maternal period. Methods: It is a descriptive study, questionnaire survey approach had been used. Pregnant mothers at all trimesters, either first, second, third and above. A sample of 150 pregnant mothers were recruited for this study by convenient sampling technique. Findings: The Mean and Standard deviation of Life stress and Emotional symptoms were 9.52, 4.63 and $11.22,4.36$ respectively. The Mean and Standard deviation of total Life stress and Emotional symptoms showed 20.27, 8.20 respectively. There was a positive correlation between Life stress and Emotional symptoms and was statistically significant at $p<0.01$. Conclusion: In our modern world this maternal stress can be maladaptive, and cause problems for the child and their family. Hence the maternal stress should be concentrated for the pregnant mother to overcome or reduce to have a healthy pregnant period, healthy baby with a healthy fetal development.

\section{KEYWORDS : Stress, Maternal Stress, Life Stress, Emotional Stress, Pregnancy.}

\section{INTRODUCTION}

Pregnancy has been viewed variously as a period of psychological stress, a normative crisis or a development phase. Pregnancy as a crisis for emotional state varies at different stages during the pregnancy depends on her capacity to adjust to physiological and psychological changes and their reactions to sexual aspects of the reproductive process influence her emotional state during pregnancy, the influence of anxiety, depression and stress in pregnancy on a variety of prenatal outcomes has been widely investigated and many literatures have been reviewed by several authors.

The Prenatal stress (or prenatal maternal stress) is exposure of an expectant mother to distress, which can be caused by stressful life events or by environmental hardships. Research indicates that prenatal stress is a significant risk factor for adverse birth outcomes, such as low birth weight and prematurity. Some women under high degrees of stress engage in risky behaviors such as alcohol and tobacco use.

Research has also shown that high levels of stress for some women can be associated with unintended pregnancy and the mother's and her partner's attitudes toward the pregnancy. The Pregnancy Risk Assessment Tracking System (PRATS) assesses prenatal stress through a 13-item inventory of stress inducing life events. These events were categorized into four groups Financial (the mother moved to a new address, her husband or partner lost his job, the mother herself lost her job, or the mother had a lot of bills she couldn't pay). Emotional (a close family member was ill and had to be hospitalized, or someone close to the mother died). Partner relationship (the mother and her husband or partner were separated or divorced, the mother experienced more arguments with her husband or partner during pregnancy than usual, or her husband or partner did not want the mother to be pregnant). Traumatic (the mother was homeless, she was involved in a physical fight, her husband or partner went to jail, or someone close to the mother had a drinking or drug problem).

"Research have also found that there is a highly significant correlation between maternal and foetal cortisol levels, although the maternal levels are ten times higher. This suggests that enough of the maternal stress hormone cortisol crosses the placenta to affect the development of the foetus." [Glover V et al]. During pregnancy these stress hormones have many other functions on top of their role in the fight or flight response. The placenta as well as the hypothalamus releases Corticotropin-releasing factor (CRF) into the bloodstream of the mother causing cortisol levels to increase. Production of Corticotropin releasing factor (CRF) by the placenta continues to rise until parturition. The levels eventually stimulate the release of prostaglandins that eventually lead to the uterine contractions that expel the baby during birth. However, studies vary substantially in terms of their methodologies and results. Some studies examine isolated stressors, such as death of a spouse or natural disaster, while others look at stressful feelings and daily hassles during pregnancy.

A women experience maternal stress in three ways that is during pregnancy, at the time of delivery and after delivery. My preference was to focus on assessment of maternal stress during pregnancy as it influences on the physiological and psychological development of the child. It's a very important period to be taken more care which has been neglected nowadays. Hence a study is conducted to evaluate the amount of maternal stress during pregnancy experienced by women.

\section{OBJECTIVES}

The major objectives of the study are

1. To identify the prevalence of women with stress during pregnancy.

2. To correlate the life stress factors and emotional stress 
factors associated with pregnancy.

3. To associate the demographic variables with the life and emotional stress factors.

4. To prepare a module to reduce stress and motivate for healthier and happier maternal period.

\section{Materials and Methods}

A descriptive approach, questionnaire survey method has been used. This study was conducted at Integral coach factory hospital, ICF, Chennai. 150 pregnant mothers at all trimesters, either first, second, third and above were recruited based on convenient sampling technique. A questionnaire was prepared consisting of Part A with demographic details, Part B with life stress factors, Part $C$ with emotional stress factors. Part A consists of 10 items of demographic details such as current age, educational qualification, occupation of self and their husband, age including the time of puberty, marriage and pregnancy, living status, number of children at pregnancy and finally trimester at which the questionnaire is given. Two main subscales include Part B contains life stress factors consisting of 9 items, Part $\mathrm{C}$ contains emotional stress factors consisting of 9 items. A total of 28 items were obtained in the questionnaire. Total score was 18 which was further categorized as mild, moderate and severe by using a range of mild 0-6, moderate - 7-12, severe 13-18. It is a standardized tool with a reliability of $r=0.89$ using Spearman's Rho prophecy. The data was analyzed by descriptive and inferential statistics.

\section{FINDINGS}

The majority of the respondents (61) perceived moderate level of Life stress with $40.7 \%$. Most of the respondents (72) perceived severe level of Emotional symptoms with $48 \%$. The total Life stress and Emotional symptoms perceived by the respondents (63) with $42 \%$. The Mean and Standard deviation of Life stress and Emotional symptoms were 9.52, 4.63 and $11.22,4.36$ respectively. The Mean and Standard deviation of total Life stress and Emotional symptoms showed 20.27, 8.20 respectively.

There was a positive correlation between Life stress and Emotional symptoms. The calculated " $r$ " value was 0.66 which was statistically at $p<0.01$. This infers that as Life stress increases Emotional symptoms also increases for mothers.

The demographic variables Educational Qualification and Living Status were found to be statistically significant at $p<$ 0.05. Whereas for occupation (Husband) and Pregnancy gravida were found to be statistically significant at $p<0.01$.

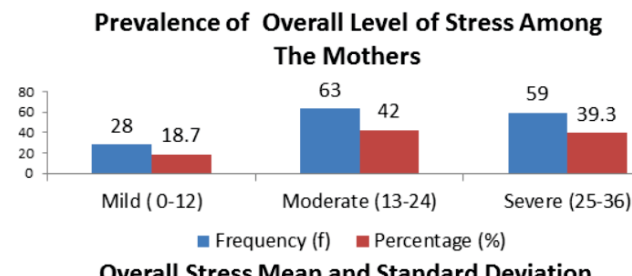

Overall Stress Mean and Standard Deviation
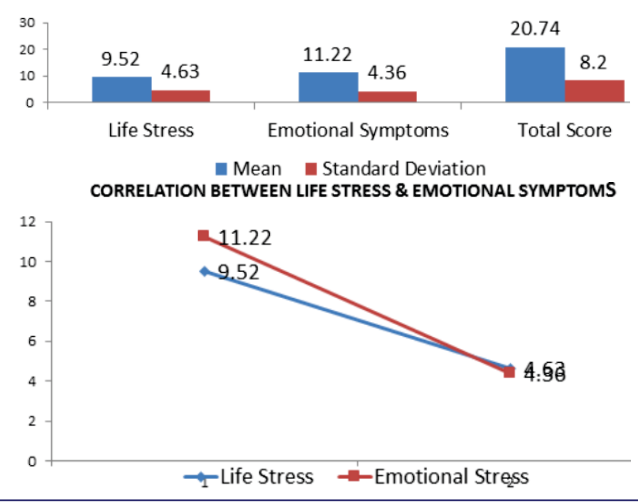

Module to reduce stress and motivate for healthier and happier maternal period.

Steps for a Healthy Pregnancy Experience

Research indicates that the growing infant in a mother's womb is more than an unfeeling organism. It actually is an active learner. Perhaps the most important aspect of prenatal parenting is creating a healthy environment for a child during pregnancy. Parents hope and dream for a healthy child, and they can help further this by learning how to avoid potential problems during pregnancy.

A healthy pregnancy and prenatal experience provide a sound foundation for a baby's growth and development and a positive parental beginning. Consider each of the areas discussed that contributes to a healthy prenatal experience.

Five critical areas to consider in creating a healthy pregnancy experience for you and your baby are:

- Manage personal health concerns

- Practice healthy behaviors

- Meet nutrition needs

- Increase your knowledge of child development

- Reduce personal stress and prepare for the child's birth

\section{MANAGE PERSONAL HEALTH CONCERNS}

Maintaining your own personal health is a key to facilitating a healthy pregnancy and a positive prenatal environment for an unborn child. Some new parents may have existing concerns they need to learn about or manage during pregnancy for a healthy prenatal experience. Some ideas to consider:

- Identify and visit a primary health-care provider early about your health needs and

- the health needs of your developing baby.

- Work to reach an appropriate weight for yourself prior to becoming pregnant if possible.

- Manage existing medical challenges.

- Find out about potential health risks.

\section{PRACTICE HEALTHY BEHAVIOURS}

Practicing healthy behaviours can give both you and your developing child a big head start in life. Try to develop a pattern of healthy living that you can carry over into your life following your pregnancy and delivery. Some ideas to consider:

- Exercise as regularly as possible during pregnancy.

- Avoid extreme diets or weight-reduction efforts.

- Avoid using alcohol, tobacco or other drugs that can have a negative impact on your child's development.

- Avoid exposure to toxic substances or chemicals.

\section{MEET NUTRITION NEEDS}

The idea that "you are what you eat" is especially true during pregnancy. A mother and her growing baby need proper nutrition to sustain growth and development. Poor nutrition can result in potential negative outcomes, such as low birth weight or a baby's premature birth. The average pregnant woman needs an additional 300 calories per day, or 2,500 to 2,700 total calories per day, depending on her size and activity level.

Some ideas to consider:

- Get adequate folic acid before and throughout pregnancy.

- Get adequate calcium vitamin D and other bone-building nutrients are found in prenatal supplements, too.

- Meet your protein needs.

- Get adequate iron. Enjoy seven or more total servings of colourful fruits and vegetables daily that provide vitamins C, A and folate, plus fibre and many others "phytochemicals"

- Meet your fluid needs.

- Take a prenatal vitamin supplement as directed by a 
health-care provider.

- Avoid alcohol during pregnancy.

- Follow safe food handling recommendations.

\section{INCREASE YOUR KNOWLEDGE OF CHILD DEVELOPMENT}

Parents' understanding and expectations of a child's growth and development significantly affect how they parent. Parents should consult with health-care providers, educators, nurses and others to learn about child development and what to expect. Some ideas to consider:

- Talk openly with your spouse or partner about what you both know and expect about a child's development.

- Read one or more basic books on the development of a child during and after pregnancy up through toddler age.

- Attend and participate in a class on childbirth that includes understanding the child's growth and needs.

- Attend and participate in a class on child development, and parenting.

- Observe other young children and talk with other parents about what they expected with a child's birth and growth versus what they experienced.

\section{REDUCE PERSONAL STRESS AND PREPARE FOR CHILD'S BIRTH}

Parents who reduce stress in their lives and prepare well for a child's birth go a long way toward creating a healthy prenatal environment. Research has shown that a stressful environment can have negative effects on a baby's development while still in the womb.

- Use relaxation techniques,

- Avoid or reduce conflict with others that may make you upset or angry.

- Practice responding to things that make you feel stressed or anxious in a calm, positive and flexible manner.

- Involve yourself in activities that are positive and make you feel good about yourself and build self-confidence.

- Take steps to involve both parents in the process of preparing for a child's birth.

- Decrease sources of stress, such as job overload or negative feelings toward others.

- Sing or hum softly to children as they grow and develop in the womb.

- Pursue activities that help you feel peaceful and reduce the strain in your life.

\section{CONCLUSION:}

Maternal stress can be chronic, linked to ongoing events in a woman's life, or acute, linked to sudden changes in a women's daily routine or environment. But in our modern world several of these changes can be maladaptive, and cause problems for the child and their family. Hence the maternal stress should be concentrated for the pregnant mother to overcome or reduce to have a healthy pregnant period, healthy baby with a healthy fetal development.

\section{REFERENCES:}

1. Biaggi, A., Conroy, S., Pawlby, S., \& Pariante, C. M. (2016). Identifying the women at risk of antenatal anxiety and depression: A systematic review. Journal of affective disorders, 191, 62-77. https:// doi. org/10. 1016/j. jad. 2015. 11.014

2. Rwakarema M, Premji SS, Nyanza EC, Riziki P, Palacios-Derflingher L. Antenatal depression is associated with pregnancy-related anxiety, partner relations, and wealth in women in Northern Tanzania: a cross-sectional study. BMC Womens Health. 2015; 15:68. Published 2015 Sep 2. doi:10.1186/s12905015-0225-y

3. Dunkel Schetter, C., \& Tanner, L. (2012). Anxiety, depression and stress in pregnancy: implications for mothers, children, research, and practice. Current opinion in psychiatry, 25(2), 141-148. https://doi. org/10. 1097/YCO. $0 \mathrm{b013}$ e 3283503680

4. Seth, S. Lewis, AJ. \& Galbally M. Perinatal maternal depression and cortisol function in pregnancy and the postpartum period: a systematic literature review. BMC Pregnancy Childbirth 16, 124 (2016)

https://doi.org/10.1186/s12884-016-0915-y

5. Glynn LM, Schetter CD, Hobel CJ, Sandman CA. Pattern of perceived stress and anxiety in pregnancy predicts preterm birth. Health Psychol. 2008;27(1):43-51. doi:10.1037/0278-6133.27.1.43
6. Woods, S. M., Melville, J. L., Guo, Y., Fan, M. Y., \& Gavin, A. (2010) Psychosocial stress during pregnancy. American journal of obstetrics and gynecology 202(1),6l.el-6l.e617. https://doi.org/10.1016/j.ajog.2009.07.041

7. Roy-Matton N, Moutquin JM, Brown C, Carrier N, Bell L. The impact of perceived maternal stress and other psychosocial risk factors on pregnancy complications. J Obstet Gynaecol Can. 2011;33(4):344-352. doi:10. 1016/s 1701-2163(16)34852-6

8. Dunkel Schetter, C., \& Tanner, L. (2012). Anxiety, depression and stress in pregnancy: implications for mothers, children, research, and practice. Current opinion in psychiatry, 25(2), 14l-148. https://doi. org/10. 1097/ YCO. 0b013e3283503680

9. Dipietro J. A. (2012). Maternal stress in pregnancy: considerations for fetal development. The Journal of adolescent health : official publication of the Society for Adolescent Medicine, 51 (2 Suppl), S3-S8. https://doi. org/10. 1016/ j. jado health. 2012.04.008

10. Baibazarova E, van de Beek C, Cohen-Kettenis PT, Buitelaar J, Shelton $\mathrm{KH}$ van Goozen SH. Influence of prenatal maternal stress, maternal plasma cortisol and cortisol in the amniotic fluid on birth outcomes and child temperament at 3 months. Psychoneuroendocrinology.2013;38(6):907-915. doi:10.1016/j.psyneuen.2012.09.015 